J. Reprod. Fert. (1967) 13, 329-331

\title{
BRIEF COMMUNICATION \\ THE PRESENCE OF TWO CONCEPTUSES IN THE UTERUS OF A NINE-BANDED ARMADILLO
}

\author{
G. DALE BUCHANAN \\ Departamento de Morfología, Facultad de Medicina, \\ Universidad del Valle, Cali, Colombia
}

(Received 3rd September 1966)

\begin{abstract}
Summary. A nine-banded armadillo was found to contain an early implantation site in the fundic tip of the uterus and an extraordinarily large free blastocyst in the central portion of the uterine cavity. This finding suggests that only the small portion of the endometrium in the fundic tip of the uterine cavity is competent to accept the blastocyst.
\end{abstract}

The nine-banded armadillo (Dasypus novemcinctus) is monovular, exhibits specific polyembryony and has a period of delayed implantation, which in the United States is about $4 \frac{1}{2}$ months long. During the delay period, the single blastocyst lies in a small depression in the endometrium at the fundic tip of the uterus (Patterson, 1913). Implantation occurs at this site, the penetrating trophoblast rapidly gaining access to the large blood sinusoids which, in this region, are quite close to the luminal epithelium (Enders, Buchanan \& Talmage, 1958).

Although implantation has not been found to occur elsewhere within the endometrium, there has been no suggestion that this very small region was physiologically different from other portions of the fundic endometrium and one could presume that implantation in this location was simply the result of the anatomic positioning of the blastocyst. Information on this point could be provided by following the fate of two blastocysts in a single uterus, but no such case appears to have been reported. In fact, in an extensive investigation of the reproductive cycle of the nine-banded armadillo begun at Rice University some years ago, several hundred female reproductive tracts were examined, and only seven cases of double ovulations were found (Enders, 1966). Three of these were non-pregnant, three had single conceptuses and one had a morula in each oviduct. Recently, the writer examined an armadillo uterus which contained two conceptuses, and is described below.

A female nine-banded armadillo was obtained from Cartago, Colombia, on 13th April 1966, and kept in the laboratory until 20th May, when it was killed. Gross examination of the reproductive tract revealed luteal tissue in the left ovary. The uterus was slightly swollen, moderately hyperaemic and probably pregnant. Upon opening the uterus an extraordinarily large blastocyst with a 
diameter of $1300 \mu$ was found in the lumen about midway between the fundus and the cervix. In addition, an implantation site containing four embryos at the embryonic shield stage was found in the fundic tip of the uterus. Except for its large size, the blastocyst appeared normal and histological examination revealed numerous mitoses in the trophoblast. The origin of the two conceptuses is enigmatic since complete sectioning of both ovaries disclosed the presence of only a single mass of luteal tissue. It is possible that the follicle which ruptured contained two ova, although such an occurrence is rare. Alternatively, two adjacent follicles may have ruptured and subsequently fused into a single luteal body, but there was no histological evidence of fusion having occurred. Lastly, the 'extra' blastocyst could be the product of a prior ovulation, subsequent to which implantation failed, but, again, this possibility could not be verified. Regardless of its origin, the unimplanted blastocyst certainly appears to have been viable and to have undergone pre-implantation changes.

The data suggest that, in the armadillo, implantation is restricted to the very small endometrial depression in the fundic tip of the uterus. This depression is formed by the intersection of two grooves, one running dorso-ventrally and the other coursing laterally between the tubo-uterine junctions. In the fundic portion of the uterus, the stromal region between the luminal epithelium and the sinusoids is reduced in thickness. Consequently, the glands have no neck region and the sinusoids are much closer to the luminal epthelium than elsewhere (Enders et al., 1958). However, neither histological nor histochemical differences have been noted in the cruciform depression compared to the adjacent portions of the endometrium.

A possible explanation for the depression being the site of implantation can be offered on morphological grounds. The space within the depression is, in effect, a cone with the apex directed toward the sinusoidal layer. Should there be two blastocysts in the depression, only one could occupy the apex of the cone, the other being necessarily less deeply situated. If implantation involves a passage of material between the blastocyst and the blood similar to that which Böving (1963) has described for the rabbit, the blastocyst in the apical position, in consequence of its being closer to the sinusoids, should be able to attach sooner than the other blastocyst. Once formed, the implantation site would establish a decidual reaction which would block implantation in the surrounding portions of the endometrium.

Perhaps as interesting as the failure of the second blastocyst to implant was its ability to grow beyond the time of nidation and achieve a diameter of three times that reported for implanting blastocysts (300 to $500 \mu$; Enders, 1963). Rapid expansion of the blastocyst precedes implantation in a number of mammals with central or eccentric implantation (e.g. carnivores, rabbit, armadillo) and can be said to trigger implantation by placing the trophoblast into intimate relation with the uterine epithelium. It has been suggested that blastocyst expansion is due to imbibition of fluid, but in this specimen the presence of numerous mitotic figures indicates that growth of the trophoblast had also occurred.

Some inference as to the ultimate fate of the blastocyst can be gained from the results of experiments with ferrets which were ovariectomized 4 to 6 days 
before implantation (Buchanan, unpublished). The endometrium remained normal, at least histologically, until some 2 weeks after the expected time of implantation and even underwent decidual changes. However, the blastocysts did not implant and died after about a week, although many underwent expansion and often became extraordinarily large, up to ten times the preimplantation diameter. While blastocyst expansion in forms such as the armadillo and ferret is apparently a prerequisite for implantation, it appears not to be an integral part of the implantation process. It is suggested that in response to some signal, perhaps humoral, the blastocyst enters an accelerated growth phase which terminates either when the blastocyst implants or outstrips its nutritional supply and perishes.

This work was supported by Grant HD-00504 from the United States Public Health Service.

\section{REFERENCES}

Böving, B. G. (1963) Implantation mechanisms. In: Conference on Physiological Mechanisms Concerned with Conception. Ed. C. G. Hartman. Pergamon Press, New York.

ENDERs, A. C. (1963) Fine structural studies of implantation in the armadillo. In: Delayed Implantation. Ed. A. C. Enders. University of Chicago Press, Chicago.

ENDERs, A. C. (1966) The reproductive cycle of the nine-banded armadillo (Dasypus novemcinctus). In: Comparative Biology of Reproduction in Mammals. Ed. I. W. Rowlands. Academic Press, London.

Enders, A. C., Buchanan, G. D. \& Talmage, R. V. (1958) Histological and histochemical observations on the armadillo uterus during the delayed and post-implantation periods. Anat. Rec. 130,639.

Patterson, J. T. (1913) Polyembryonic development in Tatusia novemcinctus. F. Morph. 24, 559. 